\title{
Immune Responses to Low Back Pain Risk Factors
}

\author{
Riley E. Splittstoesser MS ${ }^{\mathrm{a}, *}$, William S. Marras $\mathrm{PhD}^{\mathrm{a}}$ and Thomas M. Best, MD, $\mathrm{PhD}^{\mathrm{b}}$ \\ ${ }^{a}$ Department of Integrated Systems Engineering, The Ohio State University, 210 Baker Systems Engineering, 1971 \\ Neil Avenue, Columbus OH 43210, USA \\ ${ }^{\mathrm{b}}$ Department of Family Medicine, The Ohio State University, Martha Moorehouse Medical Plaza, 2050 Kenny \\ Dr., Columbus OH 43210, USA
}

\begin{abstract}
Objective: Investigate effects of interactions between biomechanical, psychosocial and individual risk factors on the body's immune inflammatory responses. Background: Current theories for low back pain causation do not fully account for the body's response to tissue loading and tissue trauma. Methods: Two groups possessing a preference for the sensor or intuitor personality trait performed repetitive lifting combined with high or low mental workload on separate occasions. Spinal loading was assessed using an EMG-assisted subject-specific biomechanical model and immune markers were collected before and after exposure. Results: Mental workload was associated with a small decrease in AP shear. Both conditions were characterized by a regulated time-dependent immune response making use of markers of inflammation, tissue trauma and muscle damage. Intuitors' creatine kinase levels were increased following low mental workload compared to that observed in Sensors with the opposite trend occurring for high mental workload. Conclusions: A temporally regulated immune response to lifting combined with mental workload exists. This response is influenced by personality and mental workload.
\end{abstract}

Keywords: Low Back Pain, Inflammation, Causation, Risk Factor Interactions

\section{Introduction}

Despite our best efforts at prevention and control, occupational low back pain (LBP) remains a common and costly condition [20]. An analysis of the Ohio Bureau of Workers' Compensation claims records ranked lumbar spine as the most common, costly of all musculoskeletal claims [8].

The National Academies have identified three primary categories of risk factors associated with occupational LBP incidence: Biomechanical, Psychosocial and Individual [20]. Biomechanical factors associated with LBP relate to the forces and motions on the lumbar spine such as load lifted, moment about L5/S1, and trunk velocity during lifting [15]. Psychosocial factors relate to the social and mental aspects of work and the body's response to them such as increased muscle loading during mental workload and changes in working postures in high pressure jobs $[2,5]$. Individual factors are conditions or be- haviors specific to the person and include anthropometry, personality and smoking status $[5,13]$.

Unfortunately, the causal pathways by which these factors contribute to LBP are poorly understood. The prevailing theory uses an engineering load-tolerance approach whereby spinal loads due to lifting is compared to the load tolerance of, typically, the L5/S1 vertebral endplates [20]. According to this model, if the load exceeds the tolerance, the tissue fails and pain results. This tolerance is usually derived from cadaver spines subject to various loading regimens and then visually inspected for structural tissue failure. However, the spine has more potential paincausing tissues than just the vertebral endplates. These include the facets, nerve roots and muscle [3].

Further, the load-tolerance model's reliance on cadaver tolerance neglects the body's biological response to loading. Recent work has shown an inflammatory response to occupational loading [25]. Inflammation is the basic process the body's tissues

\footnotetext{
* Corresponding author. E-mail: splittstoesser.1@buckeyemail.osu.edu; Phone: (614) 292-2016; Fax: (614) 292-7852
} 
use to respond to tissue trauma and initiate repair. This is a tightly regulated process as inflammation brings macrophages and other granulocytes to the site of injury. These are powerful catabolic agents that break down injured tissue to make way for healing. However, prolonged exposure can cause damage to healthy tissue as well. Therefore prolonged inflammation is almost always destructive to the body's tissues.

As inflammation does not show well in most medical imaging and the trauma required to initiate inflammation can be very small in scale, it is perhaps not surprising, then that only $15 \%$ of patients with low back pain have specific diagnoses following patient imaging [6]. This suggests a biological inflam- matory tolerance would be more appropriate than a visual tissue failure tolerance. The following model is presented to provide a biological pathway between exposure to risk factors from the national academies categories and development of LBP (Figure 1).

To elaborate on the figure, Biomechanical loading due to physical exertion traumatizes the tissues in and around the spine and has been shown to elicit an inflammatory response [25]. This inflammation causes pain and recruits neutrophils and macrophages to the site of tissue trauma. If loading ceases, inflammation resolves, the tissue heals and normal structure and function is restored. Repeated exposure before inflammatory resolution leads to chronic inflammation which causes pain, sensitizes nociceptors and

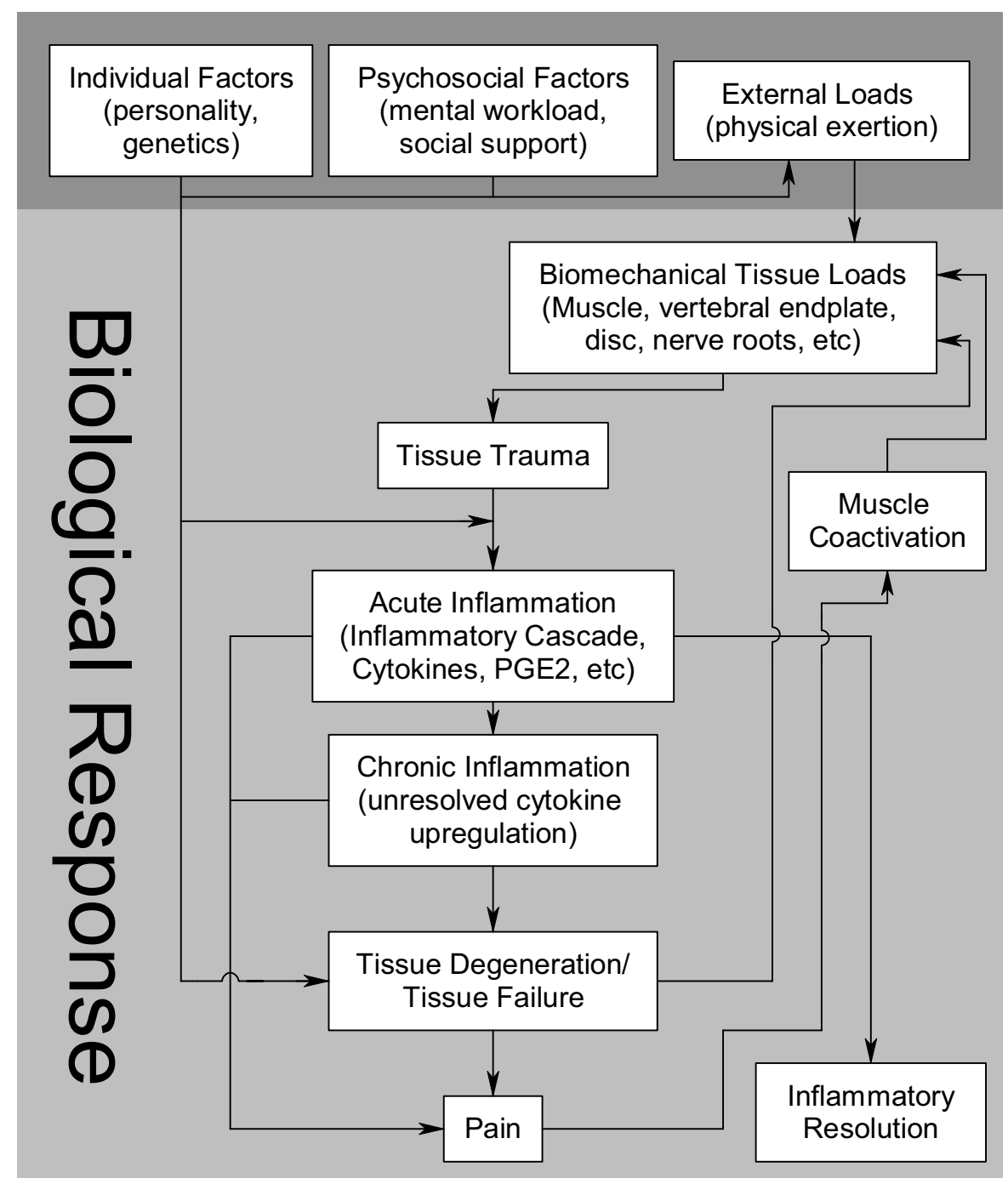

Figure 1. Theoretical model for low back pain causation 
degenerates tissue. Sufficient degeneration leads to tissue failure, pain and alterations in how tissues react to loading. In turn, LBP is associated with increased muscle coactivation during lifting which further increases biomechanical loading [17].

Psychosocial and individual factors may contribute to this inflammatory process in a number of ways. Mental load can interact with subject personality to alter an individual's lifting mechanics and, thereby, spinal loads [5]. Independently, mental stress can alter the body's inflammatory responses, and delay the healing process [12]. Of course, an individual's initial tissue tolerance will determine the tissue's ability to resist degeneration.

To investigate this process, an experiment was conducted to test the hypothesis that a biological inflammatory response exists to exposure to multiple categories of LBP risk factors.

\section{Methods}

\subsection{Approach}

An experiment was conducted to investigate the interaction between mental workload, personality and repetitive lifting on spinal loading and the body's biological responses. Subjects performed a standardized lifting task while simultaneously answering questions with different levels of complexity. Subject lifting data was fed into an Electromyographically (EMG)-assisted, subject-specific biomechanical model of the lumbar spine and used to determine spinal tissue loading. Blood and saliva samples were collected at time points before and after lifting to assess the body's biological response. At the same time points, subjects filled out discomfort surveys to determine subjective perceptions of pain.

\subsection{Study Design}

The study was a repeated measures design with two lifting sessions combined with levels of mental load and a control session with no lifting or mental load. Sessions were performed in a randomized order. Discomfort surveys, blood and saliva samples were collected at Baseline (before exposure), immediately Post Exposure, 2 Hours Post exposure and 20 Hours Post Exposure. For consistency, the experiment was run at the same time of day for all subjects so the above samples occurred at $8 \mathrm{AM}, 12$ noon, $2 \mathrm{PM}$ and $8 \mathrm{AM}$ the following day. These times were kept consistent for the control session as well. During the control session, subjects performed their normal activities of daily living but were asked to refrain from lifting or strenuous exercise. Subjects were allowed at least one week between exposures to reduce any carryover effects.

\subsection{Subjects}

20 male volunteers were recruited from the local population. All subjects reported: no history of jobs involving heavy manual materials handling in the past year, were not weight training or jogging, no history of psychiatric disorders, no musculoskeletal disorders in the past year, and no medical conditions which would alter their inflammatory response. All subjects provided informed consent. The study was approved by The University Institutional Review Board.

Table 1. Subject Anthropometry

\begin{tabular}{|l|c|c|}
\cline { 2 - 3 } \multicolumn{1}{c|}{} & Sensors & Intuitors \\
\hline Number of Subjects & 10 & 10 \\
\hline Age $(\mathrm{yrs})$ & $22.7(3.3)$ & $28.7(10.6)$ \\
\hline Weight $(\mathrm{kg})$ & $76.0(11.1)$ & $77.8(11.5)$ \\
\hline Stature $(\mathrm{cm})$ & $178.2(5.6)$ & $182.9(7.2)$ \\
\hline Hip Height $(\mathrm{cm})$ & $94.9(1.9)$ & $98.7(5.7)$ \\
\hline Acromion-Knuckle Len $(\mathrm{cm})$ & $66.2(4.2)$ & $66.8(4.2)$ \\
\hline
\end{tabular}

\subsection{Experimental Task}

Subjects performed a standardized lifting and lowering task based on previous industrial surveillance [16]. Subjects lifted a $6.8 \mathrm{~kg}$ box with handles from an origin $88 \mathrm{~cm}$ high located in front of the subject at a distance equal to that subject's acromion-knuckle length. The box was placed at a destination platform located $90^{\circ}$ clockwise at the subject's hip height and acromion-knuckle length from the midline of the body (Figure 2). Subjects lifted at the sound of a tone every 5.4 seconds with a 6 second break every 9 lifts for 2 hours.

\subsection{Apparatus}

Trunk kinematics were recorded using a Lumbar Motion Monitor (LMM) [14]. The LMM is an electrogoniometer that tracks instantaneous change in position, velocity and acceleration of the lumbar spine in three dimensional space. 


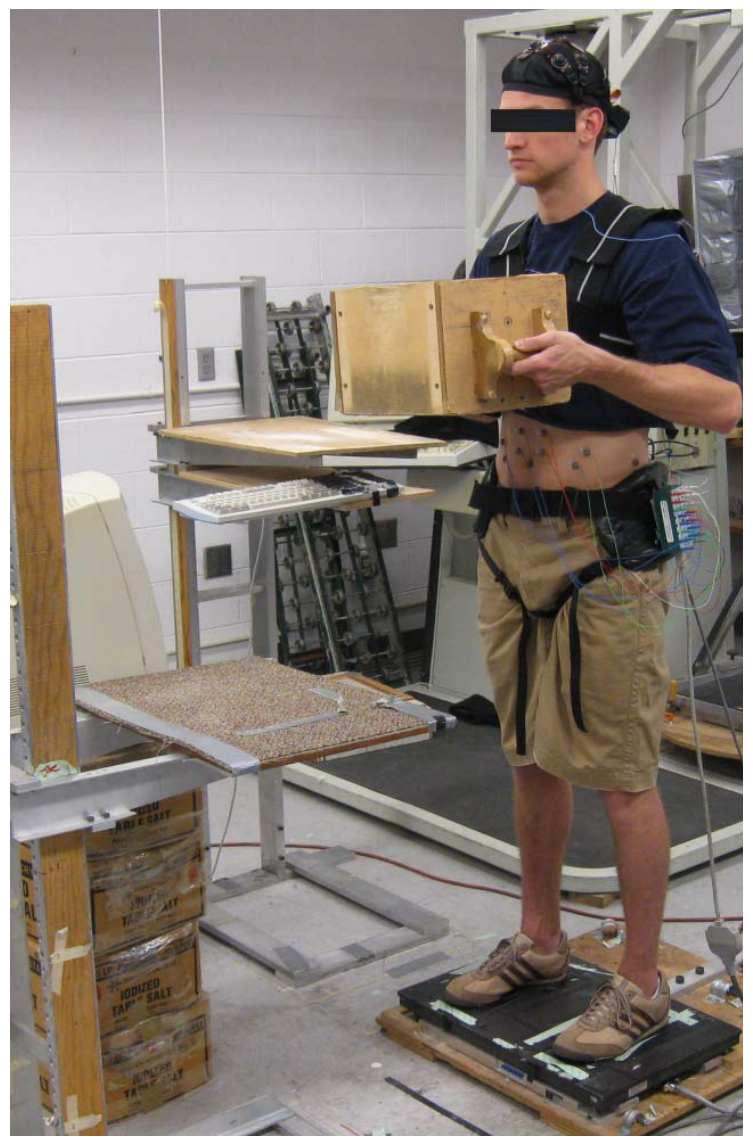

Figure 2. Experimental lifting setup

Muscle activity was collected at $1000 \mathrm{~Hz}$ using bipolar $\mathrm{Ag}-\mathrm{Cl}$ surface electrodes placed over the right and left muscle pairs for the latissimus dorsi, erector spinae, rectus abdominus, internal obliques and external obliques (Model 12 Neuradata Acquisition System; Grass Technologies, West Warwick, RI, USA). Signals were high pass filtered at $30 \mathrm{~Hz}$, low pass filtered at $1000 \mathrm{HZ}$ and notch filtered at $60 \mathrm{~Hz}$. Signals were rectified and integrated via a $20 \mathrm{~ms}$ sliding window. EMG signals were then normalized against maximum voluntary contractions preformed in each of six directions (trunk flexion, extension, right lateral, left lateral, right twist, left twist) while standing in a structure that immobilizes body in the posture needed for each exertion [11]. Electrode placement locations are detailed in the literature [18].

External forces and moments on the lumbar spine were determined using the method of Fathallah et al. 1997 [9]. A forceplate (Bertec 4060A; Bertec, Worthington, Ohio, USA) and two electrogoniometers were used to determine forces and moments continuously and dynamically throughout each exertion.
Signals were collected using custom data collection software and processed using an EMG-assisted, subject-specific biomechanical model

\subsection{Experimental Procedure}

Blood draws were performed at the Clinical Research Center (CRC) at The Ohio State University. To minimize any cortisol expression due to fear of the blood draws, saliva was collected while subjects were in transit to the CRC. To account for variability between visits a blood draw, saliva collection and discomfort surveys were performed at $8 \mathrm{AM}$ each day of the experiment. The blood draws were performed fasting and subjects were provided breakfast following the draw. For lifting exposure days, subjects were transported to the Biodynamics Laboratory where surface electrodes were placed. Each subject then performed maximum voluntary contractions within a rigid structure so trial data could be normalized. Subjects were then fitted with a LMM and given instructions on how to perform the experimental task.

Subjects stood on a forceplate while lifting and 9 lifts were collected every 10 minutes. After collecting these lifts, subject blood pressure was collected to verify any stress responses. The lifting exposure lasted for two hours.

Immediately after the lifting exposure, subjects were given discomfort surveys and transported to the $\mathrm{CRC}$ for the Post exposure saliva collection and blood draw after which subjects were provided with lunch. Additional discomfort surveys, saliva collections and blood draws were performed 2 hours after exposure and 20 hours after exposure (8AM the next day).

\subsection{Independent Variables}

Mental load (low, high) and subject Sensor or Intuitor preference as determined by the Myers-Briggs Type Indicator (MBTI) were investigated [19].

\subsubsection{Mental Load}

During clockwise lifts, the tone was accompanied by an experimental question displayed on a computer monitor next to the lift origin. Subjects were to think about the answer to the question during the lift and press a key corresponding to the answer after setting the box down. During the Low Mental Load Visit, an up or down arrow appeared on the screen with subjects instructed to press the corresponding arrow at the destination (Figure 3, Low Mental Load). During 
the High Mental Load Visit subjects were presented with a modified Stroop Color-Word task [23]. The Stroop color-word task is a mentally demanding task commonly used to induce experimental mental stress. The task presents the subject with a word that is a color (such as the word white) presented in a font color different from the color meaning of the word (Figure 2, High Mental Load). The subject was instructed to choose the answer corresponding to font color. Visually we read words before processing color so the task adds mental load by requiring subjects to discard their first impression. To add complexity, the response buttons were arranged perpendicularly to the arrangement of the response keys. Thus, the task was intended to provide high mental load compared to the low mental load task.

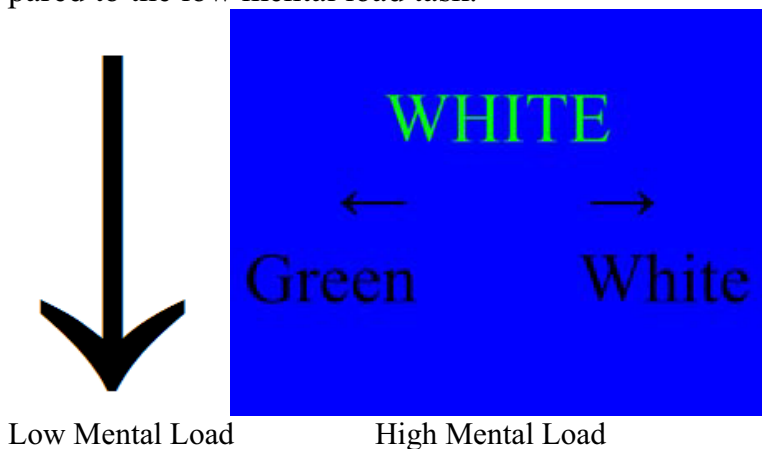

Figure 3. Samples of computer-displayed tasks

\subsubsection{Myers-Briggs Type Indicator}

Subjects in this study were selected based on their MBTI Sensor/Intuitor preference so that 10 sensors and 10 intuitors participated. The MBTI classifies subject personalities by their preferences in how they make decisions. There are four categorical preference scales: Introvert/Extravert, Sensor/Intuitor, Thinker/Feeler and Perceiver/Judger. Sensing and Intuition indicates the preferred mode of perceiving and gathering information. Sensors prefer established work routines whereas Intuitors dislike repetition and prefer to learn new skills [1]. The Sensor-Intuitor subscale rates subjects' Sensor or Intuitor preference as Slight, Moderate, Clear or Very Clear. To clearly separate preferences, subjects who were rated as slight Sensor or Intuitors were not used in this study.

\subsection{Dependent Variables}

Dependent variables were modeled spinal loads and immune response markers. Spinal loads consisted of compression, lateral and anterior-posterior shear force at the L5/S1 lumbar motion segments.
Immune markers consisted of blood and saliva levels of white blood cells (WBC), lymphocytes, granulocytes, IL-1, TNF- $\alpha$, IL-6, IL-10, Substance P, Creatine Kinase (CK) and cortisol.

\subsubsection{Biomechanical model}

EMG, kinetic and kinematic data collected during lifting was used in an EMG-assisted subject-specific biomechanical model that has been in development at the Biodynamics Laboratory for over 25 years [24]. The model provides loads for the intervertebral joints and the tissues of the lumbar spine under realistic lifting conditions that are specific to the individual subject. The model has been extensively validated.

\subsubsection{Biological sampling}

Venous blood samples were collected and white blood cell counts and $\mathrm{CK}$ concentrations were assessed immediately. For the remaining blood, serum was separated from cells and platelets by centrifugation and placed in $\mathrm{a}-80^{\circ} \mathrm{C}$ freezer until final analysis. Commercially available Enzyme Linked Immunosorbant Assay (ELISA) (MesoScale Gaithersburg, MA) kits were used to quantify circulating levels of cytokines.

Sweating, dehydration and drinking of fluids can alter plasma volume and influence measurement of plasma protein concentrations. To account for this, the method of Dill and Costill 1974 was used to correct concentrations so that all time points can be directly compared [7].

Salivary cortisol was collected using a cotton braid placed in the subject's mouth (Cortisol Coat-A-Count RIA kit, Siemens Medical Solutions Diagnostics, Los Angeles, CA, USA) for 1-2 minutes. Upon removal, the sample was placed in a sterile salivette container, centrifuged and stored for analysis.

\subsubsection{Visual Analogue Discomfort Scores}

Discomfort over parts of the entire body was recorded using a series of visual analogue scales (VAS) for the right and left neck, chest, abdomen, upper back, lower back, buttock, shoulder, upper arm, elbow, lower arm, wrist, hand, hip, upper leg, knee, lower leg, ankle, and foot. The entire body was covered to allow us to investigate relationships between specific painful regions and the body's biological response while being able to account for confounding from pain in other regions of the body. 


\subsection{Data Analysis}

For the biological variables, data from each subject's control session was used to remove any diurnal variation. The variables were then expressed as

\section{Results}

\subsection{Biological Responses}

Levels of IL-6, total white blood cell count, the percentage of WBCs that were granulocytes, IL-8, creatine kinase and Cortisol varied with mental load, time and the interaction between personality and mental load (Table 2). change from the Baseline for the Post, $2 \mathrm{Hr}$ Post and $20 \mathrm{Hr}$ Post time points for that session. ANOVA analyses of means and standard deviations were performed (SAS, SAS Institute Inc., Cary, NC, US).

Table 2

\section{Discussion}

The results in Figure 4 indicate a time-regulated biological response to repetitive lifting. The biological agents active in this time sequence deserve further explanation. IL-6 for example, can have pro-and antiinflammatory effects. Petersen and Pedersen have dubbed IL-6 a myokine because it can be expressed directly from the muscle during exercise rather than always as a response to tissue trauma [21]. In that

P-values of the effects of Mental Load, Time and the interaction between Personality and Mental Load on biological response.

\begin{tabular}{|c|c|c|c|c|c|c|}
\hline & IL-6 & WBC & $\%$ Granulocytes & IL-8 & $\mathrm{CK}$ & Cortisol \\
\hline Mental Load & 0.1513 & 0.1453 & 0.5943 & 0.7477 & 0.3089 & 0.0211 \\
\hline Time & 0.0008 & 0.0001 & $<.0001$ & 0.0466 & 0.0009 & 0.36 \\
\hline Personality*Mental Load & 0.871 & 0.9827 & 0.3706 & 0.9943 & 0.0361 & 0.2807 \\
\hline
\end{tabular}

The time course of IL-6, the percentage of WBCs that were granulocytes, IL-8 and CK are shown in Figure 4. IL-6 peaked immediately following exposure, followed by \% Granulocytes 2 and IL- 8 hours after exposure and CK 20 hours after exposure.

In addition, $\mathrm{CK}$ showed an interaction between personality and mental load (Figure 5). Sensors responded more strongly than Intuitors to the lifting and low mental load condition whereas the treand reverses for lifting and high mental load condition.

\subsection{Spinal Load Responses}

Spinal loading differed between mental load conditions only for anterior posterior shear $(\mathrm{p}=0.0143)$. Compared to the low mental load condition, the high mental load resulted in $11.0 \%$ less AP shear. acute role, IL-6 is anti-inflammatory in that it inhibits production of the potent pro-inflammatory cytokines $\mathrm{TNF} \alpha$ and IL-1. However, in conditions of chronic exposure or tissue injury Gabay argues that continual IL-6 exposure has a pro-inflammatory effects [10]. Prolonged IL-6 exposure alters the granulocytes at the site of tissue trauma from the general responding neutrophils to macrophages that produce IL-1.

In the context of the exposures in this study, it is likely that much of the IL- 6 upregulation observed is due to exercise. However, the elevated levels of CK indicate muscle trauma and therefore raises the possibility that some of the observed IL- 6 expression is due to tissue trauma. In either case, IL-6 remained above baseline at 8AM the day following exposure, allowing for potential prolonged IL-6 upregulation following repeated work exposures. IL-6 levels were also correlated with reported discomfort.

Two hours following exposure the \% of the total white blood cells that were granulocytes peaked. This represents a shift towards the innate immune system activity. The innate immune system responds to tissue trauma as well as non-specific pathogens in the 


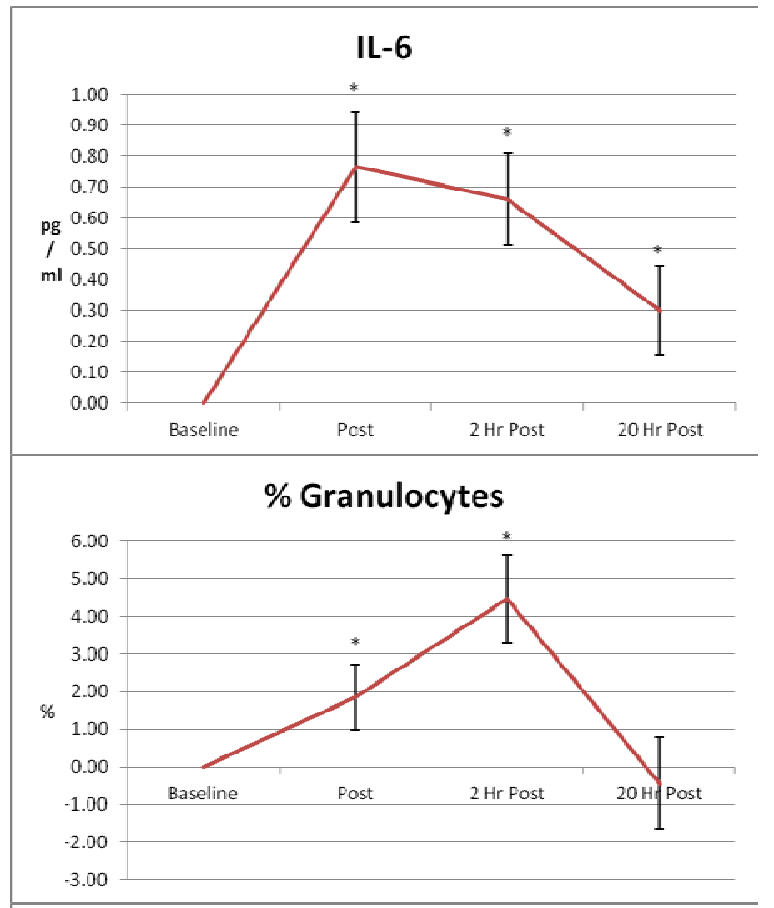

IL-8
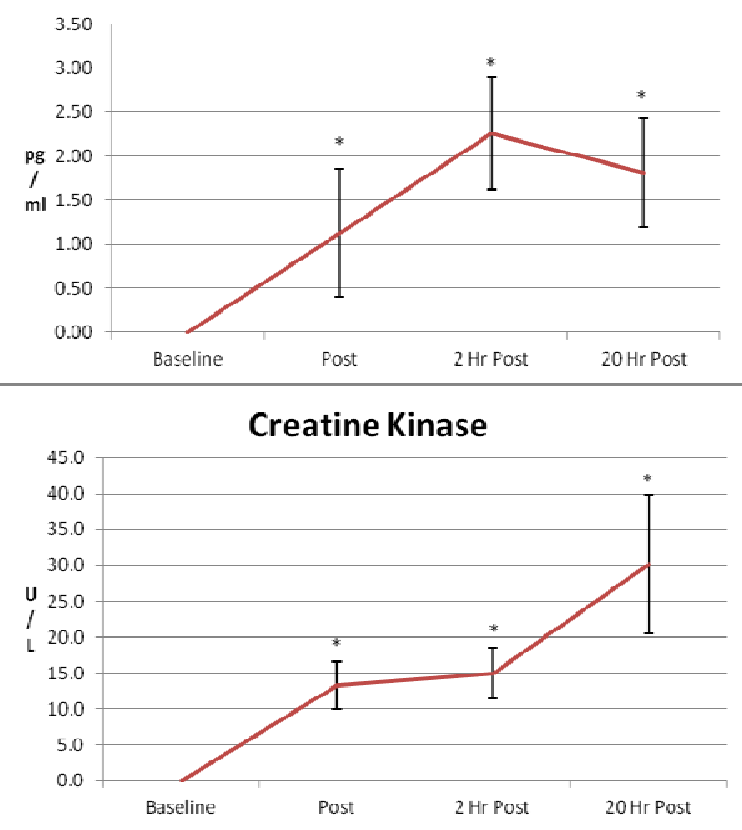

Figure 4. Changes in biological markers following experimental exposure relative to baseline.

body. Neutrophils are the most populous granulocytes in the blood and can live up to 5.4 days [22]. Thus, the return of $\%$ granulocytes to baseline 20 hours post exposure likely indicates the granulocytes have left the circulating blood that was being sampled

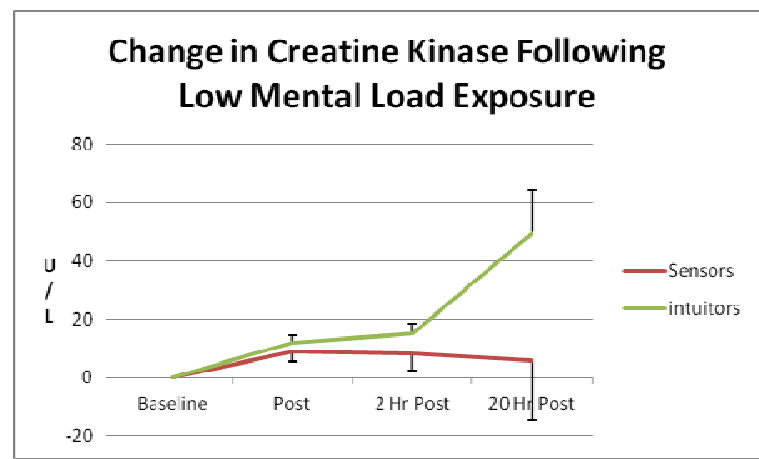

\section{Change in Creatine Kinase Following High Mental Load Exposure}

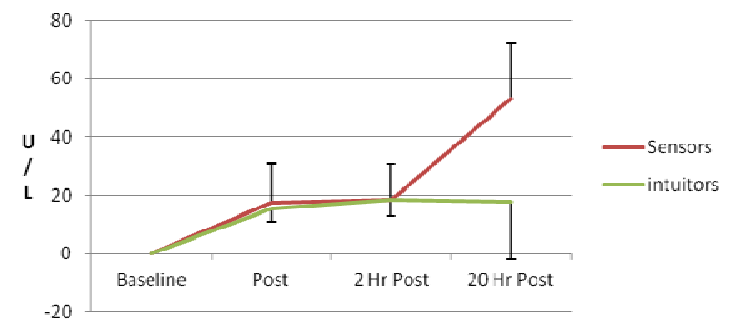

Figure 5. Changes in creatine kinase levels following low and high mental load and lifting exposures relative to baseline.

during this experiment and have migrated to the sites of tissue trauma.

The IL-8 results also support this hypothesis. IL-89 is a chemokine that serves to draw neutrophils to sites of injury. Neutrophils follow the increasing IL-8 chemical gradient to the site of injury where they carry out phagocytosis of damaged tissue. In this study, IL-8 levels in the circulating blood peaked and fell off in a similar manner to the $\%$ granulocytes.

Clinically, elevated creatine kinase levels are often used as an indicator of damage to muscle. $\mathrm{CK}$ is stored within the muscle and leaks into the interstitial fluid following muscle damage. Over time, CK then makes its way into the blood where it may be observed. Typically, studies monitoring CK use quite severe exposures such as maximum voluntary eccentric contraction regimens repeated over 6 days [4]. Therefore, the clinical significance of prolonged CK elevations like those observed in this study is unexplored.

The personality*mental load interaction observed for $\mathrm{CK}$ in Figure $\mathrm{x}$ follows an apparent mismatch between the mental load condition and subject Sensor/Intuitor preference. Intuitors dislike repetition and prefer to learn new skills whereas sensors prefer established work routines. This may explain the differ- 
ent CK responses following the Low Mental Load condition as this condition may have been perceived as more repetitious by the Intuitors and more routine by the Sensors. This CK increase occurs in the absence of a corresponding spinal load increase. Therefore, it appears CK has a biological pathway dependent on mental loading and personality The nature of that pathway is, however, unclear.

\section{Limitations}

The lifting duration in this study was limited to 2 hours with a 20 hour follow-up. It is expected that the immune effects observed here will only increase with increased exposure.

\section{Conclusions}

An immune response to lifting and mental loading tempered by personality was observed. Twenty hours post exposure, this response was not fully resolved. Following repeated exposure over multiple working days, these responses have implications for the potential low back heath of workers.

\section{Acknowledgements}

The project described was supported by Award Number UL1RR025755 from the National Center for Research Resources. The content is solely the responsibility of the authors and does not necessarily represent the official views of the National Center for Research Resources or the National Institutes of Health.

\section{References}

[1] Allread, W.G., An Investigation of the Relationship Between Personality and Risk Factors for Musculoskeletal Disorders, in Department of Industrial and Systems Engineering. 2000, The Ohio State University: Columbus. p. 151.

[2] Carayon, P., Smith, M.J., Haims, M.C., Work Organization, Job Stress, and Work-Related Musculoskeletal Disorders. Human Factors, 1999. 41(4): p. 644-663.

[3] Cavanaugh, J.M., A.C. Ozaktay, T. Yamashita, A. Avramov, T.V. Getchell, and A.I. King, Mechanisms of low back pain: a neurophysiologic and neuroanatomic study. Clin Orthop Relat Res, 1997(335): p. 166-80.

[4] Chen, T.C. and S.S. Hsieh, Effects of a 7-day eccentric training period on muscle damage and inflammation. Med Sci Sports Exerc, 2001. 33(10): p. 1732-8.

[5] Davis, K.G. and W.S. Marras, Partitioning the contributing role of biomechanical, psychosocial, and individual risk factors in the development of spine loads. Spine J, 2003. 3(5): p. 331-8.
[6] Deyo, R.A. and J.N. Weinstein, Low back pain. N Engl J Med, 2001. 344(5): p. 363-70.

[7] Dill, D.B. and D.L. Costill, Calculation of percentage changes in volumes of blood, plasma, and red cells in dehydration. J Appl Physiol, 1974. 37(2): p. 247-8.

[8] Dunning, K.K., K.G. Davis, C. Cook, S.E. Kotowski, C. Hamrick, G. Jewell, and J. Lockey, Costs by industry and diagnosis among musculoskeletal claims in a state workers compensation system: 1999-2004. Am J Ind Med, 2010. 53(3): p. $276-84$.

[9] Fathallah, F.A., W.S. Marras, M. Parnianpour, and K.P. Granata, A method for measuring external spinal loads during unconstrained free-dynamic lifting. J Biomech, 1997. 30(9).

[10] Gabay, C., Interleukin-6 and chronic inflammation. Arthritis Res Ther, 2006. 8 Suppl 2: p. S3.

[11] Granata, K.P., W.S. Marras, and F.A. Fathallah, A method for measuring external loads during dynamic lifting exertions. J Biomech, 1996. 29(9): p. 1219-22.

[12] Kiecolt-Glaser, J.K., T.J. Loving, J.R. Stowell, W.B. Malarkey, S. Lemeshow, S.L. Dickinson, and R. Glaser, Hostile marital interactions, proinflammatory cytokine production, and wound healing. Arch Gen Psychiatry, 2005. 62(12): p. 1377-84.

[13] Leboeuf-Yde, C., Back pain--individual and genetic factors. J Electromyogr Kinesiol, 2004. 14(1): p. 129-33.

[14] Marras, W.S., F.A. Fathallah, R.J. Miller, K.G. Davis, and G.A. Mirka, Accuracy of a three-dimensional lumbar motion monitor for recording dynamic trunk motion characteristics. IJIE, 1992. 9: p. 75-87.

[15] Marras, W.S., S.A. Lavender, S.E. Leurgans, S.L. Rajulu, W.G. Allread, F.A. Fathallah, and S.A. Ferguson, The role of dynamic three-dimensional trunk motion in occupationallyrelated low back disorders. The effects of workplace factors, trunk position, and trunk motion characteristics on risk of injury. Spine, 1993. 18(5): p. 617-28.

[16] Marras, W.S., S.A. Lavender, S.E. Leurgans, F.A. Fathallah, S.A. Ferguson, W.G. Allread, and S.L. Rajulu, Biomechanical risk factors for occupationally related low back disorders. Ergonomics, 1995. 38(2): p. 377-410.

[17] Marras, W.S., S.A. Ferguson, D. Burr, K.G. Davis, and P. Gupta, Spine loading in patients with low back pain during asymmetric lifting exertions. Spine J, 2004. 4(1): p. 64-75.

[18] Mirka, G.A. and W.S. Marras, A stochastic model of trunk muscle coactivation during trunk bending. Spine, 1993. 18(11): p. $1396-409$.

[19] Myers, P.B. and K.D. Myers, Myers-Briggs type indicator form. 1998: Palo Alto, California: Consulting Psychologists Press, Inc.

[20] NAS/IOM, Musculoskeletal disorders and the workplace: low back and upper extremities. 2001, Washington, DC: National Academies Press.

[21] Petersen, A.M. and B.K. Pedersen, The role of IL-6 in mediating the anti-inflammatory effects of exercise. J Physiol Pharmacol, 2006. 57 Suppl 10: p. 43-51.

[22] Pillay, J., I. den Braber, N. Vrisekoop, L.M. Kwast, R.J. de Boer, J.A. Borghans, K. Tesselaar, and L. Koenderman, In vivo labeling with $2 \mathrm{H} 2 \mathrm{O}$ reveals a human neutrophil lifespan of 5.4 days. Blood, 2010. 116(4): p. 625-7.

[23] Stroop, J., Studies of interference in serial verbal reactions. J Experimental Psychol, 1935. 18: p. 643-662.

[24] Theado, E.W., G.G. Knapik, and W.S. Marras, Modification of an EMG-assisted biomechanical model for pushing and pulling. IJIE, 2007. 37(11-12): p. 7.

[25] Yang, G., W.S. Marras, and T.M. Best, The biochemical response to biomechanical tissue loading on the low back during physical work exposure. Clin Biomech (Bristol, Avon), 2011. 26(5): p. 431-7. 